\title{
A SURVEY ON THE BLOW UP TECHNIQUE
}

\author{
MARÍA JESÚS ÁLVAREZ* \\ Departament de Matemàtiques i Informàtica, Universitat de les Illes \\ Balears; Crtra. Valldemossa, km 7.5, 07122, Palma, Spain \\ E-mail: chus.alvarez@uib.es \\ ANTONI FERRAGUT! \\ Departament de Matemàtica Aplicada I, Universitat Politècnica de \\ Catalunya; Av. Diagonal, 647, 08028, Barcelona, Catalunya, Spain \\ E-mail: antoni.ferragut@upc.edu \\ XAVIER JARQUE ${ }^{\ddagger}$ \\ Departament d'Enginyeria Informàtica $i$ Matemàtiques, Universitat \\ Rovira i Virgili; Av. Països Catalans, 26, 43007, Tarragona, Catalunya, \\ Spain \\ E-mail: xavier.jarque@ub.edu
}

The blow up technique is widely used in desingularization of degenerate singular points of planar vector fields. In this survey we give an overview of the different types of blow up and we illustrate them with many examples for better understanding. Moreover, we introduce a new generalization of the classical blow up.

\section{Introduction}

The study of the topological behavior of the solutions of a real planar vector field $X=P \partial_{x}+Q \partial_{y}$ in a neighborhood of a singular point is one of the main unsolved problems in the qualitative theory of differential systems. Concerning the simple singular points (where both eigenvalues of the jacobian matrix at the singular point are different from zero) the Hartman-Grobman Theorem completely classifies them (except the center-focus points). The semi-simple points (with one of the

\footnotetext{
${ }^{*}$ Corresponding author. Partially supported by grant MTM2008-03437.

${ }^{\dagger}$ Partially supported by grants MTM2008-03437, Juan de la Cierva, 2009SGR-410 and MTM2009-14163-C02-02

${ }^{\ddagger}$ Partially supported by grants 2009SGR-792, MTM200605849 and MTM-2008-01486 Consolider (including a FEDER contribution).
}

eigenvalues equal to zero) are also classified (see [Andronov et al., 1973]). Precisely, the local phase portrait in these two cases is either monodromic (thus a focus or a center) or a saddle, or a node, or a saddle-node.

Regarding the degenerate singular points, with both eigenvalues of the jacobian matrix at the point equal to zero, the situation is far more complicated. The topology around a non-monodromic singular point can be much richer. The Andreev Theorem (see [Andreev, 1958]) classifies the nilpotent singular points, degenerate singular points whose associated jacobian matrix is not identically zero, except the center-focus case; see also [Arrowsmith, 1979]. If the jacobian matrix is identically null (the socalled linearly zero case) the problem is open. In this case, the only possibility is studying each degenerate singular point case by case. 
The main technique to perform the desingulariztion of linearly zero singular points is the blow up technique. The desingularization theorem for planar vector fields was first stated by Bendixson in 1901 without proof. Seidenberg gave the first rigorous proof of the theorem for the analytic case, see [Seidenberg, 1968]. The desingularization procedure was extended to $\mathcal{C}^{\infty}$ vector fields of Łojasiewicz type in [Dumortier, 1977]. Van den Essen found a transformed proof of the desingularization theorem for analytic vector fields, see [Van den Essen, 1979].

Roughly speaking the idea behind the blow up technique is to explode, through a change of variables that is not a diffeomorphism, the singularity to a line or to a circle. Then, for studying the original singular point one studies the new singular points that appear on this line or circle and that will be, probably, simpler. If some of these new singular points are degenerate the process is repeated. Dumortier proved that this iterative process of desingularization is finite, see [Dumortier, 1977].

The quasi-homogeneous blow up is a widely used extension of the blow up technique. It was already used by Lyapunov but was essentially put forward as a systematic and more powerful technique in [Brunella \& Miari, 1990] and especially in [Bruno, 1989]. A proof of the desingularization theorem for $\mathcal{C}^{\infty}$ vector fields of Łojasiewicz type, based on quasi-homogeneous blow-ups, was given by Pelletier in her thesis [Pelletier, 1994]; see also [Pelletier, 1995]. The desingularization procedure using quasi-homogeneous blow ups has also been applied in the program P4 (see [Artés et al., 2005] and [Dumortier \& Herssens, 1999]).

The main goal of this work is to widely explain the blow up technique and its extensions. We shall define and explain the different types of blow up appearing in the literature and we shall provide many examples with coloured figures for better understanding. Moreover we shall introduce a new extension of the classical blow up technique, the $k$-blow up, which is very useful in some cases to reduce the number of computations.

We refer the reader to [Mañosa, 2002] for more information about blow ups in the center-focus case and to [Takens, 1974] for the rest of singularities.

\section{The homogeneous blow up technique}

Consider a real planar polynomial differential system of the form

$$
\begin{aligned}
& \dot{x}=P(x, y)=P_{m}(x, y)+\cdots, \\
& \dot{y}=Q(x, y)=Q_{m}(x, y)+\cdots,
\end{aligned}
$$

where $P$ and $Q$ are coprime polynomials, $P_{m}$ and $Q_{m}$ are homogeneous polynomials of degree $m \in \mathbb{N}$ and the dots mean higher order terms in $x$ and $y$. We note that we are assuming that the origin is a singular point, since $m>0$. Taking polar coordinates $(x, y) \mapsto(r, \theta)$, system (1) becomes

$$
\begin{aligned}
& \dot{r}=\mathcal{R}(\theta) r+\cdots, \\
& \dot{\theta}=\mathcal{F}(\theta)+\cdots,
\end{aligned}
$$

where $\mathcal{R}$ and $\mathcal{F}$ are polynomials in $\cos \theta$ and $\sin \theta$ and the dots mean higher order terms in $r$. If $\mathcal{F} \not \equiv 0$ we say that the origin is a non-dicritical singular point. In this case, all the solution curves tending to the origin in forward or backward time are tangent to the solutions $\theta^{*} \in[0,2 \pi)$ of the equation $\mathcal{F}(\theta)=$ 0 . We call $\mathcal{F}$ the characteristic polynomial of (1) at the origin and $\theta^{*}$ a characteristic direction. In cartesian coordinates $\mathcal{F}$ writes as

$$
\mathcal{F}(x, y):=x Q_{m}(x, y)-y P_{m}(x, y) .
$$

If $\mathcal{F} \equiv 0$ the origin is a dicritical singular point. In this case we easily deduce from (3) that $P_{m}=$ $x W_{m-1}$ and $Q_{m}=y W_{m-1}$, where $W_{m-1} \not \equiv 0$ is a homogeneous polynomial of degree $m-1$. If $y-v x$ is a factor of $W_{m-1}$ and $v=\tan \theta^{*}, \theta^{*} \in[0,2 \pi)$, then $\theta^{*}$ is a singular direction.

The homogeneous polar blow up (or polar blow up) is the mapping $(r, \theta) \mapsto(r \cos \theta, r \sin \theta)=$ $(x, y)$, with $r \in \mathbb{R}$ and $\theta \in[0,2 \pi)$. This map transforms the origin of system (1) into the circle $r=0$, which is called the exceptional divisor (see figure 1). After the polar blow up and after cancelling an appearing common factor $r^{m-1}$, system (1) becomes system (2). If $\mathcal{F} \equiv 0$ then this common factor is $r^{m}$.

The homogeneous directional blow up in the $x$ direction (or the directional blow up in the $x$ direction) (resp. $y)$ is the mapping $(x, z) \mapsto(x, x z)=$ $(x, y)(\operatorname{resp} .(z, y) \mapsto(y z, y)=(x, y))$, where $z$ is a new variable. This map transforms the origin of (1) into the line $x=0$ (resp. $y=0$ ), which is called the 
exceptional divisor (see figure 2). The expression of system (1) after the blow up in the $x$ direction is

$$
\dot{x}=P(x, x z), \quad \dot{z}=\frac{Q(x, x z)-z P(x, x z)}{x},
$$

that is always well-defined since we are assuming that the origin is a singularity. After the blow up, we cancel an appearing common factor $x^{m-1}\left(x^{m}\right.$ if $\mathcal{F} \equiv 0)$. Moreover, the mapping swaps the second and the third quadrants in the $x$-directional blow up and the third and the fourth quadrants in the $y$-directional blow up, which writes as

$$
\dot{z}=\frac{P(y z, y)-z Q(y z, y)}{y}, \quad \dot{x}=P(y z, y) .
$$

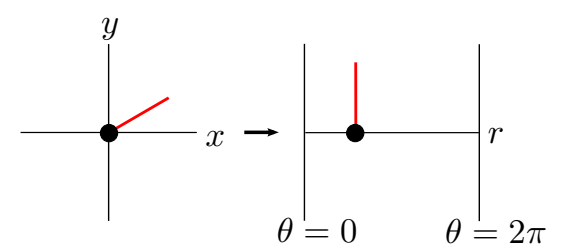

Fig. 1. The polar blow up: from the plane to the cylinder.

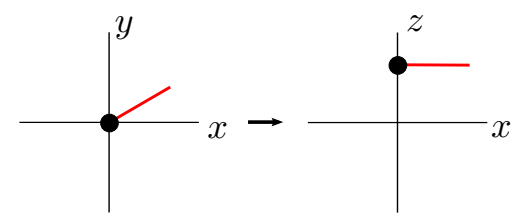

Fig. 2. The directional blow up.

The polar and the $x$-directional blow ups are equivalent on $\{x \neq 0\}(\theta \neq \pi / 2,3 \pi / 2)$, since there exists an analytic change of variables $\varphi$ bringing $(r, \theta)$ to $(x, y)$ :

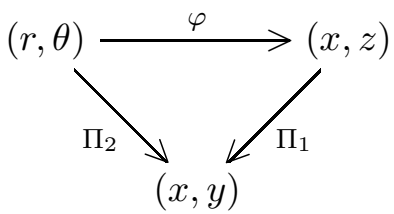

where $\varphi(r, \theta)=(r \cos \theta, \tan \theta)=(x, z), \Pi_{2}(r, \theta)=$ $(r \cos \theta, r \sin \theta)=(x, y)$ is the polar blow up and $\Pi_{1}(x, z)=(x, y)$ is the $x$-directional blow up. In the case of the $y$-directional blow up something analogous happens on $\{y \neq 0\}(\theta \neq 0, \pi)$. In practical, one uses the directional blow up rather than the polar one, as the computations and the appearing expressions are easier.
During the explanation, some of the results that we will state will be refered to the $x$-directional blow up. The reader may note that the results will be also valid for the $y$-directional blow up.

We reproduce in the following two wellknown results that provide the relationship between the original singular point of system (1) and the new singularities of system (4); see [Andronov et al., 1973].

Proposition 2.1. Let $\varphi_{t}=(x(t), y(t))$ be a trajectory tending to the origin of system (1), in forward of backward time. Suppose that $\mathcal{F} \not \equiv 0$. Assume that $\varphi_{t}$ is tangent to one of the two angle directions $\tan \theta=v, v \neq \infty$. Then the following statements hold.

(i) The two angle directions $\theta=\arctan v$ (in $[0,2 \pi)$ ) are characteristic directions.

(ii) The point $(0, v)$ on the $(x, z)$-plane is an isolated singular point of system (4).

(iii) The trajectory $\varphi_{t}$ corresponds to a solution of system (4) tending to the singular point $(0, v)$.

(iv) Conversely, any solution of system (4) tending to the singular point $(0, v)$ on the $(x, z)$ plane corresponds to a solution of system (1) tending to the origin in one of the two angle directions $\tan \theta=v$.

Proposition 2.2. Consider system (1) and suppose that $\mathcal{F} \equiv 0$. Then for every non-singular direction $\theta$ there exists exactly one semipath tending to the origin in the direction $\theta$ in forward or backward time. If $\theta^{*}$ is a singular direction, there may be either no semipaths tending to the origin in the direction $\theta^{*}$, or a finite number, or infinitely many.

The conclusion of the previous propositions is that in order to study the behavior of the solutions around the origin of system (1) it is necessary to study the singular points of system (4) on the exceptional divisor. They correspond to either characteristic directions in the non-dicritical case, or singular directions in the dicritical case. It may happen that some of these singular points are degenerate. If this is the case, then we have to study them repeating the process. As we already pointed out, it is proved 
in [Dumortier, 1977] that this chain of blow ups is finite.

We illustrate the idea of the blow up technique with some examples.

Example 2.3. Consider the planar system

$$
\begin{aligned}
& \dot{x}=a x^{2}-2 x y+\cdots, \\
& \dot{y}=y^{2}-a x y+\cdots,
\end{aligned}
$$

with $a \in \mathbb{R}^{+}$, where the dots mean higher order terms in $x$ and $y$. We study the local behavior of this system around the origin, which is a degenerate singular point. We shall desingularize it with both polar and directional blow up.

The polar blow up transforms system (6) into

$$
\begin{aligned}
\dot{r}= & \left(a \cos ^{3} \theta+\sin ^{3} \theta-2 \cos ^{2} \theta \sin \theta\right. \\
& \left.-a \cos \theta \sin ^{2} \theta\right) r+\cdots, \\
\dot{\theta}= & \cos \theta \sin \theta(3 \sin \theta-2 a \cos \theta)+\cdots,
\end{aligned}
$$

where the dots mean now higher order terms in $r$. In order to desingularize the origin of system (6) we have to study the singular points of system (7) on the exceptional divisor $r=0$, which are the solutions of the equation

$$
\cos \theta \sin \theta(3 \sin \theta-2 a \cos \theta)=0,
$$

that is $0, \pi / 2, \pi, 3 \pi / 2, \arctan (2 a / 3), \arctan (2 a / 3)+$ $\pi$. All of them are hyperbolic saddles. The phase portrait of system (7) on the cylinder is shown in figure $3(\mathrm{a})$. If we close the cylinder identifying $\theta=0$ and $\theta=2 \pi$ then we see the exceptional divisor as a circle, see figure $3(\mathrm{~b})$. This is the usual way to visualize $r=0$ in the blown up system for the polar blow up. In this case, the interior of the circle represents $r<0$ and the exterior $r>0$. Consequently, we will be interested exclusively in the separatrices at the exterior of the circle.

Finally, going back through the blow up we recover the phase portrait of the original system (6) in figure 3(c). As we can see, this is easily done by shrinking the circle to a point.

Next we use the directional blow ups to desingularize the origin of system (6). The characteristic polynomial of this system is $\mathcal{F}(x, y)=x y(3 y-2 a x)$. Therefore we need to do both directional blow ups to get the complete local phase portrait around the

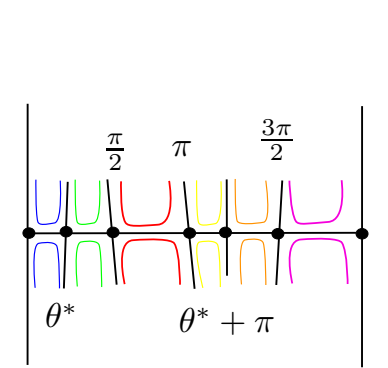

(a)

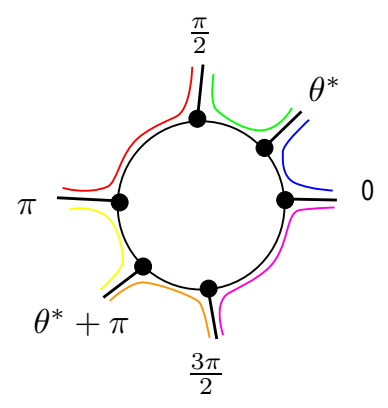

(b)

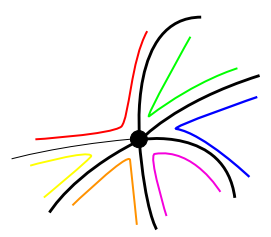

(c)

Fig. 3. The desingularization of example 2.3 using polar blow ups.

origin, as $x=0$ and $y=0$ are both characteristic directions. We start with the $x$-directional blow up $(x, y) \mapsto(x, x z)$. System (6) writes in the new variables as

$$
\begin{aligned}
& \dot{x}=x(a-2 z)+\cdots, \\
& \dot{z}=z(3 z-2 a)+\cdots,
\end{aligned}
$$

where a common factor $x$ has been cancelled. We are interested in the singular points lying on the exceptional divisor $x=0$, which are $(0,0)$ and $\left(0, \frac{2 a}{3}\right)$. Both are saddle points. The phase portrait of system (8) is shown in figure 4(a).

Next we apply the $y$-directional blow up $(x, y) \mapsto(y w, y)$, obtaining the system

$$
\begin{aligned}
\dot{w} & =w(2 a w-3)+\cdots, \\
\dot{y} & =y(1-a w)+\cdots,
\end{aligned}
$$

where a common factor $y$ has been cancelled. In this case we only need to study whether the origin is a singular point and its local behavior, as other singular points on the exceptional divisor $y=0$ different from the origin are in correspondance with the ones obtained in the $x$-directional blow up different from the origin. The origin of system (9) is also a saddle point. The phase portrait of system (9) is shown in figure 4(b).

Joining both directional blow ups one gets the local phase portrait of the original system (6) in 
figure 3(c). It is obviously topologically the same as the one obtained using the polar blow up. The exceptional divisor shrinks to a point, and hence the orbits are slightly modified. Following proposition 2.1 , the singular point $(0,2 a / 3)$ of system (8) is transformed into a solution arriving to the origin of system (6) with slope $2 a / 3$. The other solutions behave in the same way.

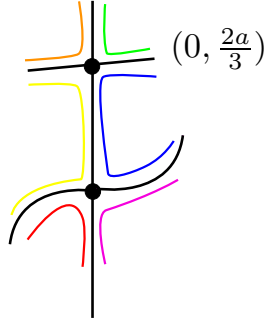

(a)

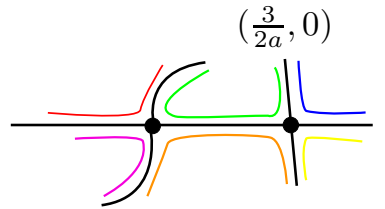

(b)

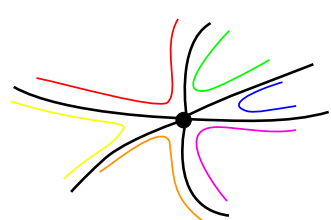

(c)

Fig. 4. The desingularization of example 2.3 using directional blow ups.

Example 2.4 (A dicritical singular point).

Consider the polynomial diffrential system appearing in [Andronov et al., 1973]

$$
\begin{aligned}
& \dot{x}=x y+(x+y)^{4}, \\
& \dot{y}=y^{2}-(x+y)^{4} .
\end{aligned}
$$

The origin is the only singular point of this system, and it is dicritical: $\mathcal{F}=x Q_{2}-y P_{2}=x y^{2}-y x y \equiv 0$. For this system, $m=2$ and $W_{1}=y$. Hence the only singular direction is $y=0$, which needs a blow up in order to be studied. There are orbits tending to the origin (in forward or backward time) in all directions different from $y=0$ (the non-singular directions), which will provide the line of singularities $x=0$ after the blow up.

After the change $y=x z$ and after dividing by $x^{2}$, system (10) becomes

$$
\begin{aligned}
& \dot{x}=z+x^{2}(1+z)^{4}, \\
& \dot{z}=-x(1+z)^{5} .
\end{aligned}
$$

The origin is the only singular point of this system. It has eigenvalues $\pm i$, hence it is either a focus or a centre. Indeed it is a centre as the system has a first integral (see [Andronov et al., 1973]). The local phase portrait of system (11) is shown in figure 5(a). In figure 5(b) we have added the line of singularities $x=0$. The orbits in figure 5 (a) become in 5(b) orbits starting and ending at two different singular points on $x=0$. Figure 5(c) shows the initial system (10): the orbits of system (11) (figure 5(b)) form two elliptic sectors close to the origin of system (10).

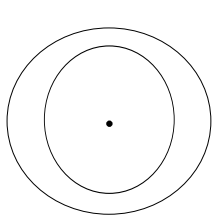

(a)



(b)

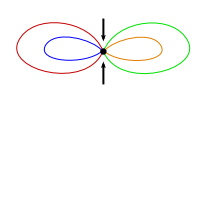

(c)
Fig. 5. The desingularization of example 2.4. The vertical arrows show how all the singular points forming the straight line $x=0$ in (b) collide to the origin in (c) and how the orbits in (b) become the orbits in (c).

In the following we present two examples that are not as simple as the previous ones, as one blow up is not enough to desingularize the singular point.

Example 2.5 (The cusp). Consider the quadratic differential system

$$
\begin{aligned}
& \dot{x}=y, \\
& \dot{y}=x^{2}+x y .
\end{aligned}
$$

The origin is the only singular point of this system and it is nilpotent. Moreover $\mathcal{F}=y^{2}$, therefore we only have the characteristic direction $y=0$. If we apply the $x$-directional blow up $y=x z$ we obtain

$$
\begin{aligned}
& \dot{x}=x z, \\
& \dot{z}=x+x z-z^{2},
\end{aligned}
$$

which has the origin as its unique singular point on the line $x=0$. It is degenerate, hence we blow up again, but this time we do the change $x=z u$, as $x=0$ is a characteristic direction: indeed $\mathcal{F}=x^{2}$. We get

$$
\begin{aligned}
& \dot{u}=-u^{2}+2 u z-u^{2} z, \\
& \dot{z}=z(u-z+u z) .
\end{aligned}
$$


On $z=0$ the origin is the only singular point and it is again degenerate. The characteristic polynomial is $\mathcal{F}=u z(2 u-3 z)$. As $z$ is a simple factor of $\mathcal{F}$ we do not need to do the blow up in the $z$ direction but in the $u$ direction. After the change $z=u v$ system (14) becomes

$$
\begin{aligned}
& \dot{u}=u(-1+2 v-u v), \\
& \dot{v}=v(2-3 v+2 u v),
\end{aligned}
$$

from which we obtain two saddles at $(0,0)$ and $(0,2 / 3)$ with respective eigenvalues $-1,2$ and $-2,1 / 3$.

Hence the desingularization of the origin of system (12) is done. Next we explain how to obtain the local behavior of the origin. We have six regions in the $(u, v)$-plane. When going back to the $(u, z)$ plane these six regions may change their shape and position on the plane, as the second and the third quadrants swap from the $(u, v)$-plane; moreover the separatrice of the singular point $(0,2 / 3)$ at the $(u, v)$-plane becomes the separatrice with slope $2 / 3$ aat the $(u, z)$-plane, say $u=3 z / 2+\cdots$. In the next step the third and the fourth quadrants swap and the curve $u=3 z / 2+\cdots$ at the $(u, z)$ plane becomes the curve $z= \pm \sqrt{2 x / 3}+\cdots$ at the $(x, z)$-plane, $x \geq 0$. The straight line $z=0$ is lost as a separatrice, as it was the exceptional divisor, and we change from six regions to four. In the last step the second and the third quadrants swap, the curve $z= \pm \sqrt{2 x / 3}+\cdots$ becomes the cusp $y= \pm \sqrt{2 x / 3} x+\cdots$ and $x=0$ is lost as a separatrice. We change from four regions to two and we are finished. The local phase portraits can be seen in figure 6 .

Example 2.6. Consider the polynomial system

$$
\begin{aligned}
& \dot{x}=y^{2}+x^{7}, \\
& \dot{y}=y^{3}\left(1+x^{2}\right) .
\end{aligned}
$$

The origin is its unique singular point and it is degenerate. The characteristic polynomial at the origin is $\mathcal{F}=y^{3}$, and then $y=0$ is the only characteristic direction. We apply the $x$-directional blow up $(x, y) \mapsto(x, x z)$ to obtain (after cancelling a common factor $\left.x^{2}\right)$

$$
\begin{aligned}
& \dot{x}=x\left(x^{5}+z^{2}\right), \\
& \dot{z}=-z\left(x^{5}+z^{2}-x z^{2}-x^{3} z^{2}\right) .
\end{aligned}
$$
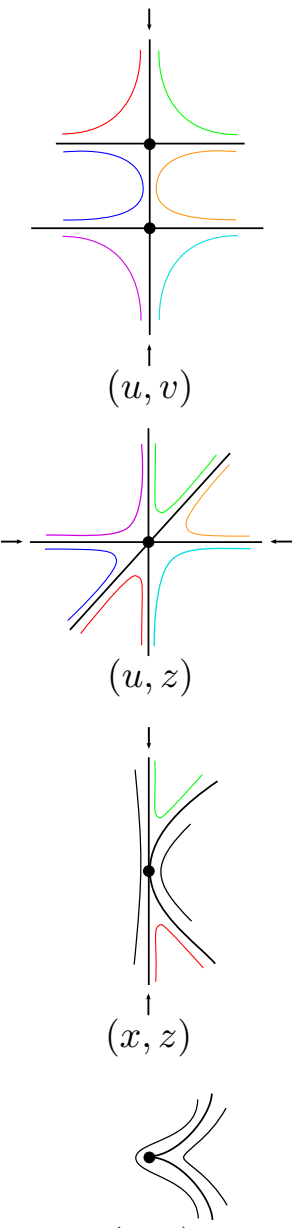

$(x, y)$

Fig. 6. The desingularization of example 2.5.

The origin is the only singular point of the new system on $x=0$ and it is also degenerate, hence we need to do another blow up. The characteristic polynomial is now $\mathcal{F}=-2 x z^{3}$. Obviously $x=0$ is a characterictic direction because it is invariant. If the factor $x$ were powered to some factor greater than one in the expression of $\mathcal{F}$, this would mean that some more orbits would arrive at the origin with vertical tangent, so we would need to do a blow up in the $y$ direction. As in this case it is simple (it is powered to one) we do not need to do the blow up in this direction. Consequently, we have to apply only the $x$-directional blow up $(x, z) \mapsto(x, x t)$, from which we get (after cancelling a common factor $x^{2}$ )

$$
\begin{aligned}
\dot{x} & =x\left(t^{2}+x^{3}\right), \\
\dot{t} & =t\left(-2 t^{2}+t^{2} x-2 x^{3}+t^{2} x^{3}\right) .
\end{aligned}
$$

The origin of this sytem is again the unique singular point on $x=0$, and it is still degenerate. Thus we 
need to apply the blow up again. The characteristic polynomial is $\mathcal{F}=-3 x t^{3}$. Again we have $x=0$ as a simple characteristic direction, thus we have to blow up the system only in the $x$ direction: $(x, t) \mapsto$ $(x, x u)$. After cancelling the common factor $x^{2}$, we obtain the new system

$$
\begin{aligned}
& \dot{x}=x\left(u^{2}+x\right), \\
& \dot{u}=u\left(u^{2} x^{3}+u^{2} x-3 u^{2}-3 x\right) .
\end{aligned}
$$

Once again, the origin is the only singular point on $x=0$ and it is degenerate. The new characteristic polynomial is $\mathcal{F}=-4 x^{2} u$, hence we have to apply now both directional blow ups, because the line $x=$ 0 is not a simple characteristic direction anymore. Thus we will apply a blow up in the $x$ direction and desingularize all the orbits arriving at the origin of (17) not tangent to $u=0$ and afterwards we will do an $u$-directional blow up to study the orbits arriving tangents to this straight line. We start with the blow up $(x, u) \mapsto(x, x v)$, obtaining the system

$$
\begin{aligned}
& \dot{x}=x\left(v^{2} x+1\right), \\
& \dot{v}=v\left(v^{2} x^{4}+v^{2} x^{2}-4 v^{2} x-4\right) .
\end{aligned}
$$

The origin is the only singular point: it is a saddle. We apply the $u$-directional blow up $(x, u) \mapsto$ $\left(u v_{1}, u\right)$ to system (17) to obtain

$$
\begin{aligned}
& \dot{v}_{1}=-v_{1}\left(u^{4} v_{1}^{3}+u^{2} v_{1}-4 u-4 v_{1}\right), \\
& \dot{u}=u\left(u^{4} v_{1}^{3}+u^{2} v_{1}-3 u-3 v_{1}\right) .
\end{aligned}
$$

The origin is a degenerate singular point, so we need to blow it up again. The characteristic polynomial is $\mathcal{F}=-7 u v_{1}\left(u+v_{1}\right)$. We have to do the blow up only in the $u$-direction because $u=0$ is a simple characteristic direction. With this blow up $\left(v_{1}, u\right) \mapsto\left(u w_{1}, u\right)$ one gets

$$
\begin{aligned}
& \dot{w}_{1}=-w_{1}\left(2 u^{6} w_{1}^{3}+2 u^{2} w_{1}-7 w_{1}-7\right), \\
& \dot{u}=u\left(u^{6} w_{1}^{3}+u^{2} w_{1}-3 w_{1}-3\right) .
\end{aligned}
$$

This system has two non-degenerate singular points: $(0,0)$, which is a saddle, and $(-1,0)$, which has one zero eigenvalue. Putting it in normal form, see [Andronov et al., 1973] we know it is a node.

Finally we go back through all the blow ups we have applied to obtain the phase portraits of all the systems until we get the phase portrait of system (16), see figure 7 .

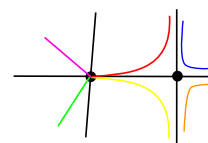

$\left(w_{1}, u\right)$
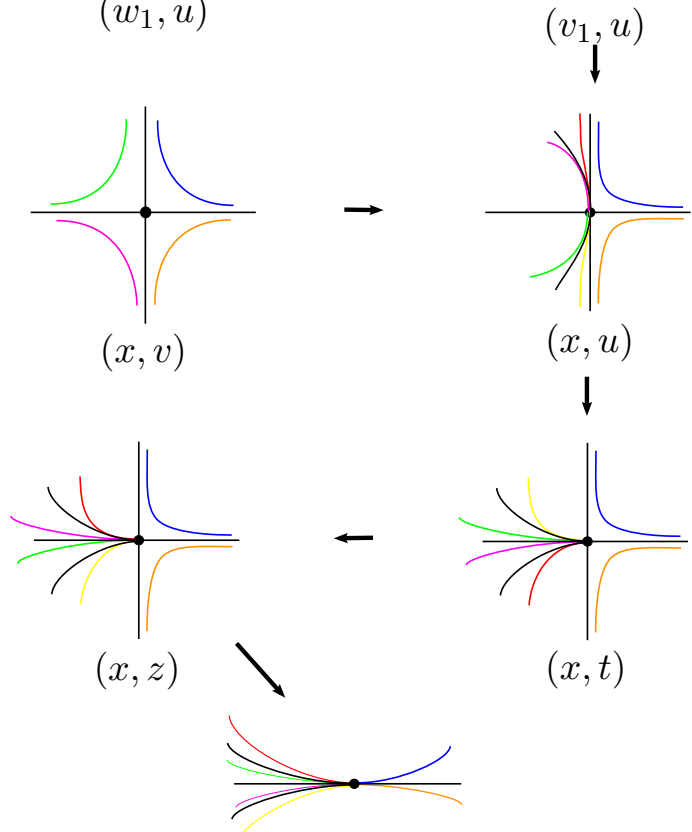

$(x, y)$

Fig. 7. The desingularization of example 2.6 .

\section{Extensions}

As we have seen in the previous examples, when desingularizing a singular point by the blow up technique a high number of computations are needed. Besides the homogeneous blow up defined in section 2 there exists in the literature the socalled $(\alpha, \beta)$-blow up or quasi-homogeneous blow up, an extension of the classical one. It is very useful as it allows to desingularize a degenerate singular point in (usually) less steps than the classical technique. The parameters $\alpha$ and $\beta$ are found from the monomials of the system, as we will show next.

\subsection{The quasi-homogeneous blow up}

The quasi-homogeneous polar blow up (or the $(\alpha, \beta)$-polar blow up) is the mapping $(r, \theta) \mapsto$ $\left(r^{\alpha} \cos \theta, r^{\beta} \sin \theta\right)=(x, y)$, with $r \in \mathbb{R}$ and $\theta \in$ $[0,2 \pi)$, for some convenient $\alpha, \beta \in \mathbb{N}$. The case $\alpha=\beta=1$ is the homogeneous polar blow up defined above.

System (1) becomes, after the quasi- 
homogeneous polar blow up,

$$
\begin{aligned}
& \dot{r}=\frac{r^{\beta} P \cos \theta+r^{\alpha} Q \sin \theta}{r^{\alpha+\beta-1}\left(\alpha \cos ^{2} \theta+\beta \sin ^{2} \theta\right)}, \\
& \dot{\theta}=\frac{\alpha r^{\alpha} Q \cos \theta-\beta r^{\beta} P \sin \theta}{r^{\alpha+\beta}\left(\alpha \cos ^{2} \theta+\beta \sin ^{2} \theta\right)},
\end{aligned}
$$

where $P$ and $Q$ are evaluated at $\left(r^{\alpha} \cos \theta, r^{\beta} \sin \theta\right)$. A common factor $r^{d}$ is to be canceled, for some suitable $d$.

The quasi-homogeneous directional blow up in the positive (resp. negative) $x$ direction is the mapping $(u, v) \mapsto\left(u^{\alpha}, u^{\beta} v\right)=(x, y)$ (resp. $\quad(u, v) \mapsto$ $\left.\left(-u^{\alpha}, u^{\beta} v\right)=(x, y)\right)$, where $u, v$ are new variables. The quasi-homogeneous directional blow up in the positive (resp. negative) y direction is the mapping $(u, v) \mapsto\left(u v^{\alpha}, v^{\beta}\right)=(x, y)$ (resp. $\quad(u, v) \mapsto$ $\left.\left(u v^{\alpha},-v^{\beta}\right)=(x, y)\right)$, where $u, v$ are new variables. The parameters $\alpha, \beta \in \mathbb{N}$ are chosen conveniently. If $\alpha$ (resp. $\beta$ ) is odd, the blow up in the positive $x$ direction (resp. $y$ direction) provides the information of the blow up in the negative $x$ direction (resp. $y$ direction). If $\alpha=\beta=1$ we recover the directional blow up defined in section 2. Moreover, if $\beta$ is odd, then the mapping swaps the second and the third quadrants in the $x$-directional blow up; and if $\alpha$ is odd then the $y$-directional blow up swaps the third and the fourth quadrants.

The expression of system (1) after the quasihomogeneous directional blow up in the $x$ direction is

$$
\dot{u}=\frac{ \pm P}{\alpha u^{\alpha-1}}, \quad \dot{v}=\frac{\alpha u^{\alpha-1} Q \mp \beta u^{\beta-1} v P}{\alpha u^{\alpha+\beta-1}},
$$

where $P$ and $Q$ are evaluated at $\left( \pm u^{\alpha}, u^{\beta} v\right)$ and the \pm means either positive or negative direction. After this blow up a common factor $u^{d}$ is to be cancelled, for some suitable $d$. Applying the quasihomogeneous directional blow up in the $y$ direction system (1) becomes

$$
\dot{u}=\frac{\beta v^{\beta-1} P \mp \alpha u v^{\alpha-1} Q}{\beta v^{\alpha+\beta-1}}, \quad \dot{v}=\frac{ \pm Q}{\beta v^{\beta-1}},
$$

where $P$ and $Q$ are evaluated at $\left(u v^{\alpha}, \pm v^{\beta}\right)$ and the \pm means either positive or negative direction. After the blow up we cancel the common factor $v^{d}$, for some suitable $d$.

Remark 3.1. As in the homogeneous blow up, the quasi-homogeneous polar blow up and the quasihomogeneous directional blow up are equivalent.
Next we show how to compute the parameters $\alpha, \beta$ and $d$, see [Pelletier, 1995] and [Bruno, 1989] for more details. We consider system (1) and write $P(x, y)=\sum_{i+j \geq m} a_{i, j} x^{i} y^{j}, \quad Q(x, y)=\sum_{i+j \geq m} b_{i, j} x^{i} y^{j}$.

We define the set

$$
N=\left\{(i-1, j): a_{i, j} \neq 0\right\} \cup\left\{(i, j-1): b_{i, j} \neq 0\right\} .
$$

The point $(-1, j)$ is associated to the monomial $a_{0, j} y^{j}$ and the point $(i,-1)$ to the monomial $b_{i, 0} x^{i}$. The point $(0,0)$ is associated to the monomials $a_{1,0} x$ and $b_{0,1} y$. If the origin of system (1) is degenerate then $(0,0) \notin N$. We define the Newton polyhedron as the convex hull of $N+\mathbb{R}_{+}^{2}$ in the $(i, j)$-plane. We call $\gamma_{k}$ the segments of this polyhedron. If one of these segments is completely in the half-plane $i \leq 0$ (resp. $j \leq 0)$ we call it $\gamma_{0}\left(\right.$ resp. $\left.\gamma_{n+1}\right)$. The rest of the segments are called $\gamma_{1}, \ldots, \gamma_{n}$ from left to right, and they have at least one endpoint in the first quadrant of the $(i, j)$-plane. For $k=1, \ldots, n$, the segment $\gamma_{k}$ satisfies the equation of the straight line $\alpha_{k} i+\beta_{k} j=\delta_{k}$, for some coprime $\alpha_{k}, \beta_{k}$ and some $\delta_{k}$. We choose the suitable $\alpha, \beta, d$ from the set $\left\{\left(\alpha_{k}, \beta_{k}, \delta_{k}\right), k=1, \ldots, n\right\}$ provided by $\gamma_{1}, \ldots, \gamma_{n}$.

If $(i, j) \in N$, then either $a_{i+1, j} x^{i+1} y^{j}$ is a monomial of $P$, or $b_{i, j+1} x^{i} y^{j+1}$ is a monomial of $Q$. We call $\delta=\alpha i+\beta j$ the quasi-degree of type $(\alpha, \beta)$ of these monomials. The monomials $a_{i+1, j} x^{i+1} y^{j}$ (resp. $b_{i, j+1} x^{i} y^{j+1}$ ) of quasi-degree $\delta$ of type $(\alpha, \beta)$ are grouped in a polynomial $P^{\delta}$ (resp. $Q^{\delta}$ ). Hence the vector field $X=(P, Q)$ can be decomposed into its quasi-homogeneous components of type $(\alpha, \beta)$ : $X=\sum_{\delta>d} X^{\delta}$, where $X^{\delta}=\left(P^{\delta}, Q^{\delta}\right)$ and $d=$ $\min \{\delta: \exists(i, j) \in N, \alpha i+\beta j=\delta\}$. We also write $X^{\gamma_{k}}:=X^{d_{k}}$ for the component of the vector field associated to the segment $\gamma_{k}$. This is a different way of writing system (1). It may be also useful to find the characteristic polynomial of system (1), which writes as

$$
\mathcal{F}=\alpha Q^{d}(1, v)-\beta v P^{d}(1, v),
$$

assuming that $\gamma: \alpha i+\beta j=d$.

When the $(\alpha, \beta)$-blow up is applied, the characteristic polynomial $\mathcal{F}$ is very useful in order to obtain the singular points of the new system that need to be studied. This polynomial is constructed from $\left(P^{d}, Q^{d}\right)$. The best parameters $(\alpha, \beta, d)$ we 
can choose in order to optimize the desingularization process are the ones provided by the segments $\gamma_{k}$.

We note that, after applying the quasihomogeneous directional blow up in the $x$ direction, only the direction $x=0$ is to be studied. This is why we also need to apply the blow up in the $y$ direction. However the following proposition, stated in [Pelletier, 1995], helps us to know whether this second blow up is to be done.

Proposition 3.2. The vertical direction $x=0$ is a characteristic direction of $X^{\gamma_{k}}$ if and only if $\gamma_{k}$ does not contain points of negative abscissa (if and only if $\left.x \mid P^{d_{k}}\right)$.

Next we apply the $(\alpha, \beta)$-blow up technique to two examples. In the first one we study again the system presented in example 2.5 to show that the $(\alpha, \beta)$-blow up is faster than the classical one. In the second one a more difficult example is provided to show the strongness of the $(\alpha, \beta)$-blow up and to better understand its application. The phase portraits in the quasi-homogeneous case are usually drawn using the polar representation, as it is, in this case, quiteclearer than the directional one.

Example 3.3 (The cusp revisited). Consider again the quadratic differential system

$$
\begin{aligned}
& \dot{x}=y, \\
& \dot{y}=x^{2}+x y,
\end{aligned}
$$

which was studied in example 2.5. We want to apply the $(\alpha, \beta)$-blow up technique in order to desingularize the singular point at the origin. In example 2.5 we have performed three directional blow ups to desingularize the origin and we will see that with the $(\alpha, \beta)$-blow up, one is enough. The set $N$ associated to system (22) is

$$
N=\{(-1,1),(2,-1),(1,0)\} .
$$

We show in figure 8 the Newton polyhedron associated to $N$. There is only one segment $\gamma_{1}$, which lies on the straight line $2 i+3 j=1$. Hence we get $\alpha=2, \beta=3$ and $d=1$.

To illustrate how the quasi-homogeneous blow up works, we appy both, the polar and the directional blow up.



Fig. 8. The Newton polyhedron of system (22).

We begin with the quasi-homogeneous polar blow up and apply the mapping $(x, y)=$ $\left(r^{2} \cos \theta, r^{3} \sin \theta\right)$. After dividing by a common factor $r$ the resulting system is

$$
\begin{aligned}
& \dot{r}=-r \sin \theta \cos \theta(r \sin \theta+\cos \theta+1), \\
& \dot{\theta}=3 \sin ^{2} \theta-2 \cos ^{3} \theta-2 r \sin \theta \cos ^{2} \theta .
\end{aligned}
$$

We compute the singular points on the exceptional divisor $r=0$ and we get that are the solutions of the equation

$$
-2 \cos ^{3} \theta+3 \sin ^{2} \theta=0 .
$$

Concretely, $\frac{\sin ^{2} \theta}{\cos ^{3} \theta}=\frac{2}{3}$. We get that these two points are saddles and the process of desingularizing is done. We can see its phase portraits in figure 9.

Next we apply the quasi-homogeneous directional blow up. In this case we have to apply a $(2,3)$-blow up in the positive $x$ direction $x=u^{2}$, $y=u^{3} v$ to obtain, after cancelling a common factor $u^{d}=u$,

$$
\begin{aligned}
& \dot{u}=\frac{u v}{2}, \\
& \dot{v}=1-\frac{3}{2} v^{2}+u v .
\end{aligned}
$$

This system has two saddles at $(0, \pm \sqrt{2 / 3})$. Applying the $(2,3)$-blow up in the negative $x$ direction $x=-u^{2}, y=u^{3} v$, we obtain

$$
\begin{aligned}
& \dot{u}=-\frac{u v}{2}, \\
& \dot{v}=1+\frac{3}{2} v^{2}-u v,
\end{aligned}
$$

which has no real singular points.

If we want to write $X=\sum_{\delta \geq 1} X^{\delta}$ we draw straight lines passing through the points of $N$ and parallel to $\gamma_{1}$. In the case of the example, besides the points $(-1,1)$ and $(2,-1)$ belonging to 
$2 i+3 j=1$ we have $(1,0)$ which belongs to $2 i+3 j=$ 2. Hence $X=X^{1}+X^{2}$, where $X^{1}=\left(y, x^{2}\right)$ and $X^{2}=(0, x y)$. As $x \nmid P^{1}, x=0$ is not a characteristic direction. Moreover we easily compute $\mathcal{F}=y^{2}$. Following proposition 3.2 we do not need to blow up in the $y$-direction.

Hence the desingularization of the origin of system $(22)$ is done and leads to the picture shown in figure 9. The cusp $y= \pm \sqrt{2 / 3} x^{3 / 2}+\cdots, x>0$, is obtained from the separatrices $v= \pm \sqrt{2 / 3}+\cdots$ of system $(23)$ at the singular points $(0, \pm \sqrt{2 / 3})$, as $v=\frac{y}{u^{3}}=\frac{y}{x^{3 / 2}}$.

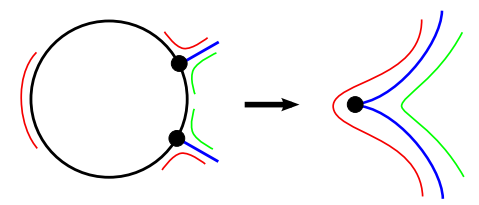

Fig. 9. The desingularization of example 3.3.

Example 3.4. Consider the polynomial vector field appearing in [Pelletier, 1995]

$$
\begin{aligned}
& \dot{x}=x^{2} y^{2}(x-y)^{2}+y^{10}, \\
& \dot{y}=x^{10} .
\end{aligned}
$$

The origin is a degenerate singular point of this system. We shall use the $(\alpha, \beta)$-blow up technique in order to desingularize this point and get the local behavior in a neighborhood of it.

Let

$$
N=\{(3,2),(2,3),(1,4),(-1,10),(10,-1)\} .
$$

The Newton polyhedron associated to $N$ is drawn in figure 10. We get three segments $\gamma_{1}, \gamma_{2}, \gamma_{3}$ which belong (respectively) to the straight lines $3 i+j=7$, $i+j=5$ and $3 i+7 j=23$. We choose the third one and apply a $(3,7)$-blow up. We have chosen this segment because we think that it illustrates better the idea of applying an $(\alpha, \beta)$-blow up, but, obviously, we could have chosen anyone of the three segments.

As $\alpha=3$ is odd it is enough to do the quasihomogeneous blow up in the $x$-direction in only one sense, and it leads to

$$
\begin{aligned}
& \dot{u}_{1}=u_{1} v_{1}^{2}\left(u_{1}^{44} v_{1}^{8}+u_{1}^{8} v_{1}^{2}-2 u_{1}^{4} v+1\right), \\
& \dot{v}_{1}=-7 u_{1}^{44} v_{1}^{11}-7 u_{1}^{8} v_{1}^{5}+14 u_{1}^{4} v_{1}^{4}-7 v_{1}^{3}+3 .
\end{aligned}
$$

This system has a unique singular point, $(0, \sqrt[3]{3 / 7})$ and it is a saddle. It is depicted in figure 14(a) in colours green $(x>0)$ and orange $(x<0)$.

Following proposition 3.2, as $\gamma_{3}$ does not contain points of negative abscissa, we have to do the blow up in the $y$ direction. We do the blow up $(x, y)=\left(u_{2} v_{2}^{3}, v_{2}^{7}\right)$ and as $\beta=7$ is also odd it is enough to do it in one sense. We get

$$
\begin{aligned}
& \dot{u}_{2}=-\frac{3 u_{2}^{11}}{7}+u_{2}^{4}-2 u_{2}^{3} v_{2}^{4}+u_{2}^{2} v_{2}^{8}+v_{2}^{44} \\
& \dot{v}_{2}=\frac{u_{2}^{10} v_{2}}{7} .
\end{aligned}
$$

As we have already done the blow up in the $x$ directions we have to pay attention only to the origin, that is degenerate.

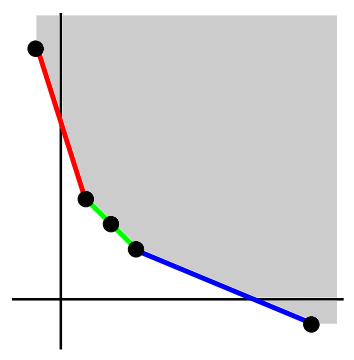

Fig. 10. The Newton polyhedron of system (25).

The Newton polyhedron associated to system (27) is constructed through the set (renaming it again as $N$ )

$$
N=\{(3,0),(10,0),(2,4),(1,8),(-1,44)\},
$$

that is drawn in figure 11. We get two significative segments $\gamma_{1}, \gamma_{2}$ which belong respectively to the straight lines $18 i+j=26$ and $4 i+j=12$. We choose the first one, $\gamma_{1}$. As it is not completely contained in the first quadrant it is not necessary to do the blow up in the $y$ direction. Consequently we apply a $(18,1)$-blow up to system $(27)$ in the positive $x$ direction and we get a new system:

$$
\begin{aligned}
& \dot{u}=7 u^{29}-3 u^{155}-14 u^{15} v^{4}+7 u\left(v^{8}+v^{44}\right), \\
& \dot{v}=-7 v\left(u^{28}-3 u^{154}-2 u^{14} v^{4}+v^{8}+v^{44}\right) .
\end{aligned}
$$

The origin is the only singular point and it is degenerate. We have to do the blow up in the negative $x$ direction, as $\alpha$ is even. In this case, the blow up we do is $\left(u_{2}, v_{2}\right)=\left(-u^{18}, u v\right)$ and the system we get is 
(which we write again in coordinates $(u, v)$ ):

$$
\begin{aligned}
& \dot{u}=-u\left(7 u^{28}+3 u^{154}+14 u^{14} v^{4}+7 v^{8}+7 v^{44}\right), \\
& \dot{v}=7 v\left(u^{28}+3 u^{154}+2 u^{14} v^{4}+v^{8}+v^{44}\right) .
\end{aligned}
$$

Thus, again the origin is the only singular point and it is degenerate. A further study shows that no relevant information is obtained from this system.

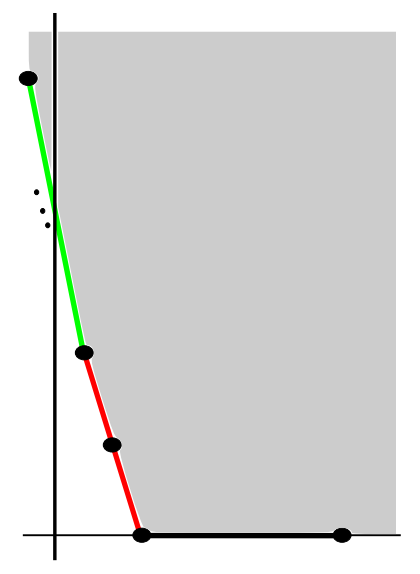

Fig. 11. The Newton polyhedron of system (27). A non-useful segment $\gamma_{3}$ is drawn in black.

Concerning the origin of system (28), we compute the set $N$ and we get

$$
N=\{(28,0),(154,0),(14,4),(0,8),(0,44)\},
$$

from which we obtain the Newton polyhedron of figure 12 . We get one significative segment $\gamma_{1}$ which belongs to the straight line $2 i+7 j=56$. Thus we apply a $(2,7)$-blow up to system $(28)$. In the positive $x$ direction we get a new system (again in coordinates $(u, v))$ :

$$
\begin{aligned}
& \dot{u}=u\left(u^{252}\left(7 v^{44}-3\right)+7\left(v^{4}-1\right)^{2}\right), \\
& \dot{v}=-63 v\left(u^{252}\left(v^{44}-1\right)+\left(v^{4}-1\right)^{2}\right) .
\end{aligned}
$$

The origin is a saddle and there are two more singular points $(0,1)$ and $(0,-1)$, both degenerate.

As $\alpha$ is even we have to do the blow up of system (28) also in the negative $x$ direction, from which we obtain exactly the same result as in the previous system (29).

Observe that in the step we are in the chain of blow ups, see figure $14(\mathrm{c})$, the singular point $(0,-1)$ is not to be studied as it is is situated in a zone of the exploded cylinder that is contained in $r<$ 0 . Consequently, we desingularize only the singular point $(0,1)$ by doing more blow ups. It is depicted in red and blue in figure 14(c).

First, we move the point to the origin. The Newton polyhedron for the new system, see in figure 13 , is constructed through the set

$N=\{(0, k): k=1, \ldots, 8\} \cup\{(252, k): k=0, \ldots, 44\}$.

We get again only one significative segment $\gamma_{1}$ that belongs to the straight line $i+252 j=252$. We do a $(1,252)$-blow up in the $x$ direction (and it is enough to do it in one sense) and the system we get is

$$
\begin{aligned}
& \dot{u}=u(4+\cdots), \\
& \dot{v}=-63 v(60+16 v+\cdots),
\end{aligned}
$$

where the dots mean higher order terms in $u$ and $v$. This system has two singular points: $(0,0)$ and $(0,-15 / 4)$, the first one being a saddle and the second a node.

Finally, the blow up in the $y$ direction provides a saddle at the origin.



Fig. 12. The Newton polyhedron of system (28)



Fig. 13. The Newton polyhedron of system (29) at $(0,1)$.

The local phase portraits of the desingularization process are shown in figure 14. Next we show how we can obtain an approximation of the separatrices in a neighborhood of the origin. In figure 14 we observe that there are two separatrices 


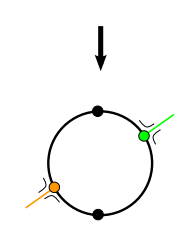

(a)



(b)

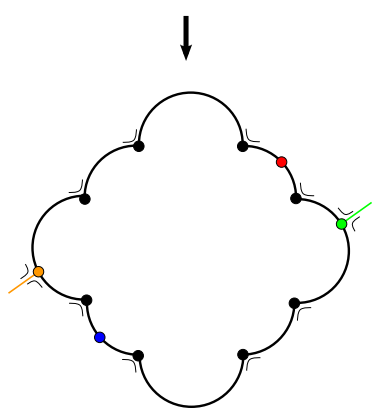

(c)

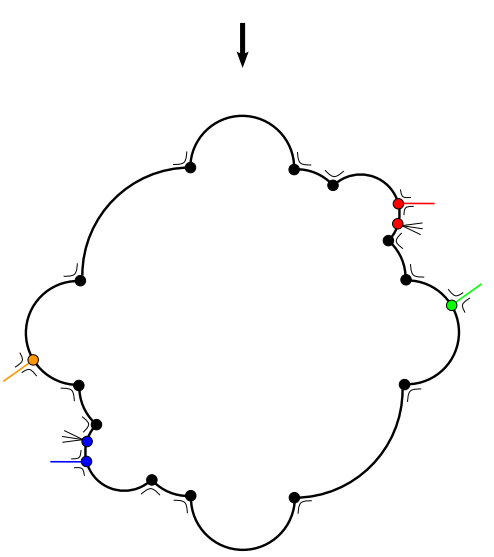

(d)

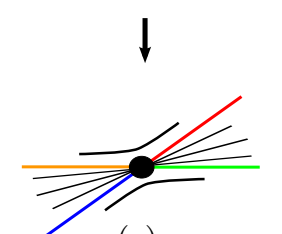

(e)

Fig. 14. The desingularization of example 3.4.

close to the origin. One of them corresponds to the singular point $(0,-15 / 4)$ appearing after the chain of blow ups $(3,7),(18,1),(2,7)$ and $(1,252)$.
Hence after these four blow ups there is a curve $v=-15 / 4+\cdots$ crossing this singular point. Going back one step means undoing the $(1,252)$-blow up. We have:

$$
\left.\begin{array}{l}
x^{252}=u^{252} \\
y=u^{252} v
\end{array}\right\} \Rightarrow \frac{y}{x^{252}}=v \Rightarrow y=\frac{-15}{4} x^{252}+\cdots,
$$

hence we obtain the corresponding separatrice before the fourth blow up. We rename it $v=$ $-15 / 4 u^{252}+\cdots$. Now, we move it to the singular point $(0,1)$ and it becomes $v=1-15 / 4 u^{252}$. Undoing the $(2,7)$-blow up we have

$$
\left.\begin{array}{l}
x^{7}=u^{14} \\
y^{2}=u^{14} v^{2}
\end{array}\right\} \Rightarrow \frac{y^{2}}{x^{7}}=v^{2} \Rightarrow y^{2}=x^{7}+\cdots .
$$

Renaming again, the separatrice at this step of the blow down is $v^{2}=u^{7}+\cdots$. Going back one more step means undoing the $(18,1)$-blow up in the $x$ direction; we have

$$
\left.\begin{array}{l}
x=u^{18} \\
y^{18}=u^{18} v^{18}
\end{array}\right\} \Rightarrow \frac{y^{18}}{x}=v^{18} \Rightarrow y^{18}=x^{9 / 2}+\cdots,
$$

as $v^{18}=u^{63}+\cdots$ and consequently, renaming again the variables we have $v^{2}=u^{1 / 2}$ or equivalently, $v^{4}=u+\cdots$. We go back through the $(3,7)$-blow up but now in the $y$ direction and we get

$$
\left.\begin{array}{l}
x^{7}=u^{7} v^{21} \\
y^{3}=v^{21}
\end{array}\right\} \Rightarrow \frac{x^{7}}{y^{3}}=u^{7} \Rightarrow x^{7}=y^{7}+\cdots .
$$

There are no more blow ups to be undone, hence we obtain an approximation of the separatrice close to the origin.

The other separatrice corresponds to the singular point $(0, \sqrt[3]{3 / 7})$ appearing after the $(3,7)$-blow up in the $x$ direction. If we undo this blow up we get

$$
\left.\begin{array}{rl}
x^{7} & =u^{21} \\
y^{3} & =u^{21} v^{3}
\end{array}\right\} \Rightarrow \frac{y^{3}}{x^{7}}=v^{3} \Rightarrow y^{3}=\frac{3}{7} x^{7}+\cdots,
$$

as $v=\sqrt[3]{3 / 7}+\cdots$. As there are no more blow ups to undone that is the approximation of the separatrice.

\subsection{The k-blow up}

We introduce in this subsection a particular case of the quasi-homogeneous directional blow up (or another generalization of the blow up), the directional 
$k$-blow up, or simply the $k$-blow up. This type of blow up allows to apply many directional blow ups (in the same direction) at once. We follow the ideas given by Álvarez and Jarque in [Álvarez, 2006].

The $k$-blow up in the $x$ direction (resp. $y$ direction) is the mapping $(x, z) \mapsto\left(x, x^{k} z\right)=(x, y)$ (resp. $\left.(z, y) \mapsto\left(y^{k} z, y\right)=(x, y)\right)$, where $z$ is a new variable. The parameter $k \in \mathbb{N}$ is chosen conveniently. If $k=1$ we recover the homogeneous directional blow up already defined. We note that the $k$-blow up is the particular case of the quasihomogeneous directional blow up when either $\alpha=1$ ( $x$ direction) or $\beta=1$ ( $y$ direction). After the $k$-blow up, a common factor $x^{d}$ is cancelled, for some suitable $d$. Moreover, if $k$ is odd the mapping swaps the second and the third quadrants in the $x$-directional blow up and the third and the fourth quadrants in the $y$-directional blow up.

After the $k$-blow up in the $x$ direction, system (1) becomes

$$
\begin{aligned}
& \dot{x}=P\left(x, x^{k} z\right), \\
& \dot{z}=\frac{Q\left(x, x^{k} z\right)-k x^{k-1} z P\left(x, x^{k} z\right)}{x^{k}} .
\end{aligned}
$$

In a similar way, after the $k$-blow up in the $y$ direction system (1) becomes

$$
\begin{aligned}
& \dot{z}=\frac{P\left(y^{k} z, y\right)-k y^{k-1} z Q\left(y^{k} z, y\right)}{y^{k}}, \\
& \dot{y}=Q\left(y^{k} z, y\right) .
\end{aligned}
$$

The suitable $k$ is the maximum natural number such that for any $k^{\prime} \in \mathbb{N}, k^{\prime}<k$, after the $k^{\prime}$-blow up the origin is the only singular point, it is degenerate and moreover the characteristic direction $x=0$ is simple. This is justified as follows:

- If other singular points appear, then they need to be studied. Moreover if the origin is not degenerate then the desingularization is over.

- If $x=0$ is not a simple characteristic direction then it may exist an orbit different from $x=0$ tending to the origin with vertical slope, and therefore at this step a blow up in the other direction is to be done.

We note that the computation of $k$ is easier than the computation of the parameters $(\alpha, \beta)$ of the quasi-homogeneous blow up, as it can be done directly from system (31) and system (32).

Remark 3.5. After applying a $k$-blow up, the singular points $(0, a)$ different from the origin correspond to orbits $y=a x^{k}+\cdots$ of the initial system.

In the following we take the differential system appearing in example 2.6 and apply the $k$-blow up technique. We will see that the gain is quite big.

Example 3.6. We study again system (16):

$$
\begin{aligned}
& \dot{x}=y^{2}+x^{7}, \\
& \dot{y}=y^{3}+x^{2} y^{3} .
\end{aligned}
$$

We recall that the origin is the unique singular point and that it is degenerate. Its characteristic polynomial is $\mathcal{F}=y^{3}$; hence $y=0$ is the only characteristic direction. Consequently we can do a $k$-blow up in the $x$-direction, $(x, y) \mapsto\left(x, x^{k} u\right)$ :

$$
\begin{aligned}
& \dot{x}=x^{2 k} u^{2}+x^{7}, \\
& \dot{u}=x^{2 k} u^{3}+x^{2 k+2} u^{3}-k x^{2 k-1} u^{3}-k x^{6} u .
\end{aligned}
$$

We have to study first which is the suitable $k$ to do the blow up. If $k=1$ then the only singular point on $x=0$ is the origin and it is degenerate; moreover $\mathcal{F}=2 x u^{3}$ and therefore $x=0$ is a simple direction. If $k=2$ then the same situation occurs, now with $\mathcal{F}=3 x u^{3}$. If $k=3$ then again the only singular point on $x=0$ is the origin and it is degenerate, but now $\mathcal{F}=4 x^{2} u$ and therefore $x=0$ is not a simple direction anymore. Hence we take $k=3$. The resulting system, after dividing by $x^{5}$, is

$$
\begin{aligned}
& \dot{x}=x\left(x+u^{2}\right), \\
& \dot{u}=u\left(x^{3} u^{2}+x u^{2}-3 x-3 u^{2}\right) .
\end{aligned}
$$

Observe that we obtain system (17) in example 2.6. At this step we have to do both directional blow ups.

We begin by doing a $k$-blow up in the $x$ direction. It turns out that the suitable blow up is the simple one, $(x, u) \mapsto(x, x v)$. We get, after dividing by $x$, the system

$$
\begin{aligned}
& \dot{x}=x\left(v^{2} x+1\right), \\
& \dot{v}=v\left(v^{2} x^{4}+v^{2} x^{2}-4 v^{2} x-4\right) .
\end{aligned}
$$

The origin is the only singular point: it is a saddle. 
We go back to system (33) and we do a $k$-blow up but now in the $u$ direction. From the two conditions stated above, it turns out that the suitable $k$ is $k=2$, i.e. we do the mapping $(x, u) \mapsto\left(u^{2} w_{1}, u\right)$. We obtain system (18):

$$
\begin{aligned}
& \dot{w}_{1}=-w_{1}\left(2 u^{6} w_{1}^{3}+2 u^{2} w_{1}-7 w_{1}-7\right), \\
& \dot{u}=u\left(u^{6} w_{1}^{3}+u^{2} w_{1}-3 w_{1}-3\right) .
\end{aligned}
$$

As we have already studied this system, we know that it has a saddle at $(0,0)$ and a node at $(-1,0)$.

We go back through the blow ups, see figure 15. Observe that most of the steps in the process that we did in example 2.6 are skipped, and the only important phase portraits are the ones that have a dynamical meaning.

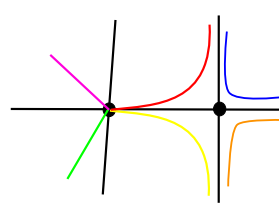

$\left(w_{1}, u\right)$



$(x, v)$
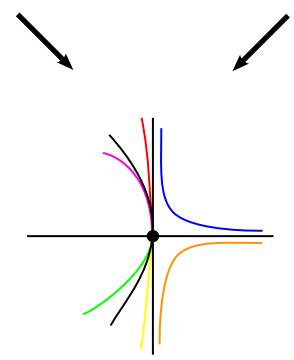

$(x, u)$



$(x, y)$

Fig. 15. The desingularization of example 3.6 by using $k$-blow ups.

\section{The integrable case}

Although the previous extensions of the blow up technique improve the method of desingularizing a degenerate singular point, this process can be still very long and can involve a high number of computations, see for instance example 3.4. Nevertheless, in the case that the system has some integrable properties, all the above mentioned computations can be avoided because all the information is obtained from the solutions of the system.

Concretely, we deal in this section with the directional homogeneous blow up technique of section 2 in the case that a rational first integral is defined in a neighborhood of the origin. We follow the results stated in [Álvarez and Ferragut, 2009]. In that paper a different point of view of the blow up technique is given, since the solutions of the system in a neighborhood of the origin are studied instead of the expression of the system itself. We use directional blow ups.

Consider the polynomial system (1). A first integral $H$ of system (1) is a $\mathcal{C}^{1}$ function such that $P H_{x}+Q H_{y}=0$, where the subindex denote partial derivative. Suppose that system (1) has a rational first integral $H=f / g$. Then the level curves $H=-c$ of the system write as $f+c g=0$, for $c \in \mathbb{C} \cup\{\infty\}$, where $c=\infty$ means $g$. Let $R=g^{2} \operatorname{gcd}\left(H_{x}, H_{y}\right)$ be the socalled remarkable factor. It is known that $R=$ $\prod u_{i}^{\alpha_{i}-1}$, where the $u_{i}$ are the so-called critical remarkable curves and the $\alpha_{i}$ are their exponents; see [Chavarriga et al., 2003] for a definition. The product runs on all the critical remarkable curves. It is proved in [Álvarez and Ferragut, 2009] that the objects defined above remain invariant after a blow up.

The following proposition allows to control whether the characteristic polynomial $\mathcal{F}$ of the blown up system is identically zero without computing the differential system explicitly. We denote by $\hat{h}$ the homogeneous polynomial of lowest degree of a polynomial function $h$ and by $m_{h}$ the degree of this homogeneous polynomial.

Proposition 4.1. We have $\mathcal{F} \equiv 0$ if and only if $m_{f+c g}=m_{g}$ for all $c \in \mathbb{C}$.

Remark 4.2. If $\mathcal{F} \not \equiv 0$ then there exists $s \in \mathbb{C} \cup\{\infty\}$ such that $m_{f+s g}>m_{f+c g}$ for all $c \in \mathbb{C} \cup\{\infty\}, c \neq s$. We assume that $s=0$ without loss of generality.

After this proposition we can discern whether 
the origin is dicritical or not. If it is dicritical, then the singular directions are to be computed. For that purpose we state the following proposition.

Proposition 4.3. Let $w$ be a homogeneous polynomial of degree 1 . Let $e_{3} \in \mathbb{N} \cup\{0\}$ be the exponent of $w$ in the factorization of $\hat{R}$. Consider the following property:

(H1) There exists $c \in \mathbb{C} \cup\{\infty\}$ such that $w$ is a multiple factor of $\hat{f}+c \hat{g}$ with multiplicity $e_{1} \in$ $\mathbb{N} \backslash\{1\}$ and $w^{e_{1}} \nmid \operatorname{gcd}(\hat{f}, \hat{g})$.

Then a divisor $w$ of $W_{m-1}$ either satisfies (H1) or $w \mid \operatorname{gcd}(\hat{f}, \hat{g})$. Conversely, let $w$ be a homogeneous polynomial of degree 1 such that either (H1) holds or $w$ divides $\operatorname{gcd}(\hat{f}, \hat{g})$ with multiplicity $e_{2} \in \mathbb{N}$. Then $w^{e} \mid W_{m-1}$ and $w^{e+1} \nmid W_{m-1}$, where $e=e_{1}-1+e_{2}-$ $e_{3}$ if (H1) holds (here $e_{2}=0$ if $w \nmid \operatorname{gcd}(\hat{f}, \hat{g})$ ) and $e=e_{2}-e_{3}$ otherwise.

Remark 4.4. Proposition 4.3 allows to compute the singular directions without computing the differential system explicitly. Moreover as a consequence of the computation, the value of $m$ appears naturally.

Once we know $m$ we can discern whether another blow up is to be applied or not.

Proposition 4.5. Suppose that the origin is a singular point of system (1) corresponding to a singular direction. If $m>1$ then the origin is degenearte. If $m=1$ and the origin is dicritical, then it is a star-node.

Next we deal with the case that the origin is not dicritical, that is $\mathcal{F} \not \equiv 0$. The following results provide the characteristic directions and the value of $m$ at the origin.

Proposition 4.6. The whole set of characteristic directions of the differential system (1) at the origin is obtained from the equation $\widehat{f g}=0$.

Remark 4.7. We can compute the singular points on $x=0$ of system (1) without computing the system itself.

Lemma 4.8. We have

$$
m_{f}+m_{g}-m_{R}=m+1 .
$$

Finally, depending on $m$ and $H$ we know which kind of singular point we are dealing with.

Proposition 4.9. Suppose that the origin is a singular point of system (1). Then:

(i) If $m>1$ then the origin is degenerate.

(ii) If $m=1$ and both $f=0$ and $g=0$ pass through the origin and $g=0$ does it not transversally to $x=0$, then it is nilpotent.

(iii) If $m=1$ and both $f=0$ and $g=0$ pass through the origin but $g=0$ does it transversally to $x=0$, then it is a node. Moreover $f+c g=0$ crosses the origin of system (1) with the slope of $g=0$, for all $c \in \mathbb{C} \backslash\{0\}$.

(iv) If $m=1$ and only $f=0$ passes through the origin, then it is a saddle. Moreover the separatrices of the origin of system (1) are contained in $f=0$.

We end this section with an example of application of this technique. We note that we only apply the directional blow up in the $x$ directon. Observe that we can always avoid $x=0$ to be a characteristic direction by a linear change of variables.

Example 4.10. Consider the rational function $H=$ $f / g$, where $f(x, y)=\left(x^{10}-y^{10}-x^{2} y^{9}\right)^{2}$ and $g(x, y)=\left(x^{2} y-2 y^{4}+2 x^{6}\right)^{6}$. We want to study the local behavior of the singular point at the origin of the polynomial differential system associated to $H$. As $g=0$ has a vertical tangent at the origin, we apply to the functions the change $x \rightarrow x+3 y$. We set $x_{0}:=x$ and $y_{0}:=y$.

We construct table 1 for better understanding of the explanation. In the columns $\mathrm{SP}_{f}$ (resp. $\mathrm{SP}_{g}$ ) we have the singular points on $x=0$ obtained from $f$ (resp. $g$ ). They are obtained applying the change $y=x z$ (or the corresponding one) to $f=0$ (resp. $g$ ), cancelling the common factor $x$ to its power and solving the equation on $x=0$. It provides solutions $z^{*}$, corresponding to the singular points $\left(0, z^{*}\right)$ of the blown up differential system. For example, after the change $x \rightarrow x+3 y, \hat{g}=0$ is equivalent to $x^{2} y+6 x y^{2}+9 y^{3}=0$. After the change $y=x z$, we obtain $x^{3}\left(z+6 z^{2}+9 z^{3}\right)=0$. Cancelling $x^{3}$ and solving the resulting equation we obtain the solutions $z=0$ (simple) and $z=-1 / 3$ (double). 


\begin{tabular}{|c|c|c|c|c|}
\hline $\mathrm{SP}_{f}$ & $\mathrm{SP}_{g}$ & $\hat{f}$ & $\hat{g}$ & $\hat{R}$ \\
\hline \hline$-\frac{1}{2}$ & $\star$ & $x_{1}^{2} l_{1}^{2}$ & $\star$ & $x_{1} l_{1}$ \\
$-\frac{1}{4}$ & $\star$ & $x_{1}^{2} l_{2}^{2}$ & $\star$ & $x_{1} l_{2}$ \\
$\star$ & 0 & $x_{1}^{2}$ & $y_{1}^{6}$ & $x_{1} y_{1}^{5}$ \\
$\star$ & $-\frac{1}{3}$ & $x_{1}^{2}$ & $x_{1}^{6}$ & $x_{1}^{6}$ \\
\hline 0 & 0 & $x_{2}^{2}$ & $y_{2}^{4} l_{3}^{6}$ & $x_{2} y_{2}^{3} l_{3}^{5}$ \\
\hline 0 & $\star$ & $x_{3}^{2}$ & $y_{3}^{8}$ & $x_{3} y_{3}^{7}$ \\
$\star$ & $-\frac{243}{2}$ & $\star$ & $y_{3}^{8} l_{4}^{6}$ & $y_{3}^{7} l_{4}^{5}$ \\
\hline
\end{tabular}

Table 1. Application of the algorithm in example 4.10. The $l_{i}$ are straight lines crossing the origin but different from the axis. In particular, $l_{3}=2 x_{2}+243 y_{2}$. The last three columns show respectively the homogeneous polynomials of lowest degree of $f, g$ and $R$ after the blow up and the corresponding singular point moved to the origin.

Three blow ups are needed to completely desingularize the singular point at the origin. From table 1 we can study all the singular points appearing in the whole blow up process:

1. First blow up, $x_{0}=x_{1}, y_{0}=x_{1} y_{1}$ :

- $(0,-1 / 2)$ and $(0,-1 / 4)$ : as $m_{R}=2$ and $m_{f}+m_{g}=4$ we have $m=1$ in both cases. Moreover, only $f=0$ passes through these points, hence they are saddles.

- $(0,0)$ : as $m_{R}=6$ and $m_{f}+m_{g}=8$ we have $m=1$. Moreover both $f=0$ and $g=0$ pass through this point and $g=0$ does it transversally, hence it is a node.

- $(0,-1 / 3)$ : as $m_{R}=6$ and $m_{f}+m_{g}=8$ we have $m=1$. Moreover, both $f=0$ and $g=0$ pass through this point and $g=0$ does it not transversally, hence the point is nilpotent and a new blow up is required.

Before the second blow up we move the point $(0,-1 / 3)$ to the origin.

2. Second blow up, $x_{1}=x_{2} y_{2}, y_{1}=y_{2}$ :

- $(0,0)$ : we have $m=2$, hence a new blow up is required.
3. Third blow up, $x_{2}=x_{3} y_{3}, y_{2}=y_{3}$ (where we take into account that now $m_{f}<m_{g}$ and the roles of $f$ and $g$ are swapped):

- $(0,0)$ : we have $m=1$. Moreover, both $f=0$ and $g=0$ pass through this point and $f=0$ does it transversally, hence it is a node.

- $(-243 / 2,0)$ : we have $m=1$. Moreover, only $g=0$ passes through this point, hence it is a saddle.

Now the desingularization process is done. Next we explain how to get the phase portrait of the initial system to end the process.

1. After the third blow up we obtain two singular points on the $\left(x_{3}, y_{3}\right)$-plane coming from the intersection of $y_{3}=0$ with the curves $x_{3}=0$ and $x_{3}=-243 / 2+\mathcal{O}\left(y_{3}\right)$, respectively.

2 . Back to the $\left(x_{2}, y_{2}\right)$-plane we study the origin. The canonical regions of the $\left(x_{3}, y_{3}\right)$ system are modified and we have swapped the third and fourth quadrants of the $\left(x_{3}, y_{3}\right)$ plane. The curve $y_{2}=0$ remains invariant, and the others become $x_{2}=0$ and $x_{2}=$ $-243 y_{2} / 2+\mathcal{O}\left(y_{2}^{2}\right)$, respectively.

3. Back to the $\left(x_{1}, y_{1}\right)$-plane and after swapping again the third and fourth quadrants, $y_{2}=0$ disappears as solution, $x_{2}=0$ becomes $x_{1}=0$ and $x_{2}=-243 y_{2} / 2+\mathcal{O}\left(y_{2}^{2}\right)$ becomes $x_{1}=-243 y_{1}^{2} / 2+\mathcal{O}\left(y_{1}^{3}\right)$. After these update we undo the change $y_{1} \rightarrow y_{1}-1 / 3$ and the singular point is now $y_{1}=-1 / 3$. There are three more non-degenerate singular points, as we found before.

4. Back to the initial system on the $\left(x_{0}, y_{0}\right)$ plane and after swapping the second and third quadrants, $x_{1}=0$ disappears as solution and only some branches of $f=0$ and $g=0$ remain as separatrices; $f=0$ provides an elliptic sector and $g=0$ a hyperbolic sector.

A diagram of the whole process is shown in figure 16. As it is shown in remark 4.2, there are curves having bigger multiplicity than the others. These curves are very important in the phase portrait as they may determine separatrices close to the singular point we are dealing with, see again figure 16. 


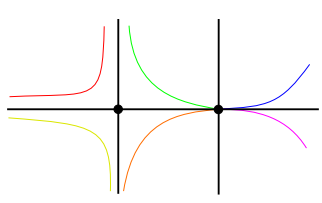

$\left(x_{3}, y_{3}\right)$

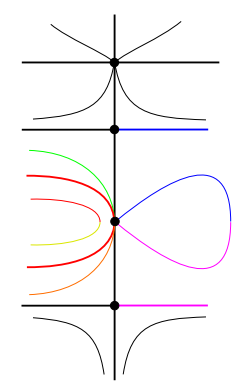

$\left(x_{1}, y_{1}\right)$

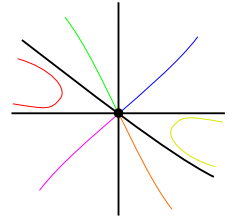

$\left(x_{2}, y_{2}\right)$

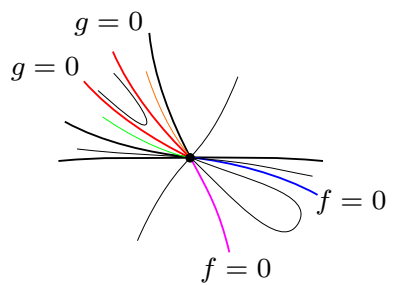

$(x, y)$
Fig. 16. The desingularization of example 4.10 .

\section{References}

Álvarez, M.J. [2006] "Critical points and periodic orbits of planar differential equations". PhD thesis, Universitat Autònoma de Barcelona.

Álvarez, M.J. \& Ferragut, A. [2009] "Local behavior of planar analytic vector fields via integrability", preprint.

Andreev, A. [1958] "Investigation on the behaviour of the integral curves of a system of two differential equations in the neighborhood of a singular point," Trans. Amer. Math. Soc. 8, 187-207.

Andronov, A.A., Leontovich, E.A., Gordon, I.I. \& Maier, A.G. [1973] "Qualitative theory of $2^{\text {nd }}$ order dynamic systems". J. Wiley \& Sons.

Arrowsmith, D.K. [1979] "The singularity $x \partial / \partial y, "$ J. Diff. Eq. 34, 153-166.

Artés, J.C., Dumortier, F., Herssens, C., Llibre, J. \& De Maesschalck, P. [2005] "Computer program P4 to study Phase Portraits of Planar Polynomial differential equations". http://mat.uab.es/artes/p4/p4.htm

Brunella, M. \& Miari, M. [1990] "Topological equivalence of a plane vector field with its principal part defined through Newton polyhedra," $J$. Diff. Eq. 85, 338-366.
Bruno, A.D. [1989] "Local methods in nonlinear differential equations". Springer Series Soviet Math. Springer.

Chavarriga, J., Giacomini, H., Giné, J. \& Llibre, J. [2003] "Darboux integrability and the inverse integrating factor," J. Diff. Eq. 194, 116-139.

Dumortier, F. [1977] "Singularities of vector fields on the plane," J. Diff. Eq. 23, 53-106.

Dumortier, F. \& Herssens, C. [1999] "Tracing Phase Portraits of Planar Polynomial Vector Fields with Detailed Analysis of the Singularities," Qual. Th. of Dyn. Sys. 1, 97-131.

Mañosa, V. [2002] "On the center problem for degenerate singular points of planar vector fields," Int. J. Bif. Chaos 12, 687-707.

Pelletier, M. [1994] "Contribution a l'étude de quelques singularités de systèmes non linéeaires". PhD thesis, Université de Bourgogne.

Pelletier, M. [1995] "Éclatements quasi homogènes," Ann. Fac. Sci. Toulouse 4, 879-937.

Seidenberg, A. [1968] "Reduction of Singularities of the Differential Equation Ady = Bdx," Amer. J. of Math. 90, 248-269.

Takens, F. [1974] "Singularities of vector fields," Publ. Math. IHES 43, 47-100.

Van den Essen, A. [1979] "Reduction of singularities of the differential equation $\mathrm{Ady}=\mathrm{Bdx}$ ". Lecture Notes in Math., 712. Springer. 